POS $\quad \begin{aligned} & \text { PROCEEDINGS } \\ & \text { OF SCIENCE }\end{aligned}$

\title{
Preface to PSTP 2015 Proceedings
}

\section{W. Meyer}

Institut für Experimentalpysik I, Ruhr-Universität Bochum, 44780 Bochum, Germany

E-mail: meyer@ep1.rub.de

\section{G. Reicherz}

Institut für Experimentalpysik I, Ruhr-Universität Bochum, 44780 Bochum, Germany

E-mail: gerhard.reicherz@rub.de

XVIth International Workshop in Polarized Sources, Targets, and Polarimetry, PSTP2015,

14-18 September 2015,

Bochum, Germany 
The 16th International Workshop on Polarized Sources, Targets and Polarimetry (PSTP 2015) was held at the Conference Center at the Ruhr-University Bochum, Germany, from September 14th -18th, 2015. This kind of workshop - focused of bringing together experts in different technical fields necessary to carry out particle physics experiments involving spin at accelerators around the world - has a long tradition (see E.Steffens, Proceedings of Science PSTP 2013, Charlottesville) since the first polarized solid target experiments in the early 1960ies performed at the accelerators in Saclay and Berkeley. The counting of the PSTP workshops started in 1988 and the Institute of Experimental Physics EP1 with its polarized solid target group is proud to act as host of the 16th workshop in this series. Sixty five scientists were registered for the workshop and forty seven talks were given. The papers from the presentations are published in these proceedings. The topics covered at the workshop were:

- Polarized Ion and Electron Sources

- Polarized Solid and Gas Targets

- Polarized Beam Polarimetry

- Applications of Spin

The Organizing Committee would like to thank all the speakers and attendees for their stimulating talks and discussions, the chairmen for their cooperation, the secretary, Daniela Klobes, for her enthusiastic help in preparing the Workshop and Dagmar Hiltscher for her very helpful assistance during the Workshop. Altogether it was a fascinating and unforgettable Workshop. Last but not least, we appreciate the financial support from the Ruhr-Universität Bochum, the Forschungszentrum Jülich, the Deutsche Forschungsgemeinschaft (TR16), the Kernphysik Institut Mainz, General Electric and Pfeiffer Vakuum. 